Christian Fandrych \& Maria Thurmair (2016):

\title{
Audioguides: Die Inszenierung von Kunst im Hörtext
}

Diese Publikation wurde zuerst veröffentlicht / This publication has been published first in:

Hausendorf, Heiko / Müller, Marcus (Hg.): Sprache in der Kunstkommunikation. Handbücher Sprachwissen. Berlin etc.: de Gruyter, 380-400. 


\section{Handbücher Sprachwissen}

Herausgegeben von

Ekkehard Felder und Andreas Gardt

\section{Band 16}




\section{Handbuch \\ Sprache in der \\ Kunstkommunikation}

Herausgegeben von

Heiko Hausendorf und Marcus Müller 
ISBN 978-3-11-029583-2

e-ISBN [PDF] 978-3-11-029627-3

e-ISBN [EPUB] 978-3-11-039506-8

Library of Congress Cataloging-in-Publication Data

A CIP catalog record for this book has been applied for at the Library of Congress.

\section{Bibliografische Information der Deutschen Nationalbibliothek}

Die Deutsche Nationalbibliothek verzeichnet diese Publikation in der Deutschen National-

bibliografie; detaillierte bibliografische Daten sind im Internet über http://dnb.dnb.de abrufbar.

(C) 2016 Walter de Gruyter GmbH, Berlin/Boston

Typesetting: fidus Publikations-Service $\mathrm{GmbH}$, Nördlingen

Printing and binding: CPI books $\mathrm{GmbH}$, Leck

@ Printed on acid-free paper

Printed in Germany

www.degruyter.com 


\section{Inhaltsverzeichnis}

Heiko Hausendorf/Marcus Müller

Sprache in der Kunstkommunikation - Einleitung — IX

I Einführung in die Thematik

Heiko Hausendorf/Marcus Müller

1. Formen und Funktionen der Sprache in der Kunstkommunikation -3

II Sprache in der Bildenden Kunst

Siegfried J. Schmidt

2. Sprache im Kunstwerk -51

Tobias Vogt

3. Sprache am Kunstwerk - 69

Martin Papenbrock/Doris Tophinke

4. Graffiti. Formen, Traditionen, Perspektiven - 88

Antje Kramer-Mallordy

5. Zwischen Revolution und Prophezeiung: Künstlermanifeste -110

III Kunstrezeption

Beate Söntgen

6. Formen der Kunstrezeption in der Moderne: Übertragung (Diderot) und Reflexion (Greenberg) — 135

Joachim Knape

7. Rhetorik des Kunstgesprächs -153

Peter Klotz

8. Ekphrasis und Kunstbeschreibung - 176

Walther Kindt

9. Kunstkommunikation und Linguistische Rhetorik — 198 


\section{Kunstkritik}

Stefan Lüddemann

10. Kunstkritik und Kunstbetrieb -225

Christiane Thim-Mabrey

11. Formen der Kunstkritik 242

\section{Kunstvermittlung}

Mechthild Dehn

12. Kunstkommunikation

in der Deutschdidaktik 265

Maria Peters

13. Sprache in der Kunstpädagogik -287

Karin-Sophie Richter-Reichenbach

14. Sprache in der Kunsttherapie -316

Swantje Karich

15. Die Kunstvermittlung im Feuilleton - 336

\section{$\mathrm{VI} \quad$ Ausstellungs- und Museumskommunikation}

Dirk vom Lehn/Christian Heath

16. Kunstinteraktion -361

Christian Fandrych/Maria Thurmair

17. Audioguides: Die Inszenierung von Kunst im Hörtext - 380

Rahel Ziethen

18. Textsorte „Ausstellungskatalog“

Patricia Harpring/Murtha Baca

19. Art Vocabulary: Categorizing Works of Art -425 


\section{Kunstwissenschaft}

Hans-Werner Eroms

20. Sprache in den Kunstwissenschaften - 455

Hubert Locher

21. Geschichte der kunsthistorischen Literatur - 479

Marcus Müller

22. Erzählen im kunstwissenschaftlichen Diskurs - 497

Register -517 


\title{
17. Audioguides: Die Inszenierung von Kunst im Hörtext
}

\begin{abstract}
Audioguides sind eine junge Textsorte im Spektrum der Ausstellungs- und Museumskommunikation mit komplexen kommunikativen Aufgaben. Im Beitrag wird gezeigt, welche sprachlichen Strategien in den Hörtexten der Audioguides eingesetzt werden, um das Kunsterlebnis der Betrachter zu verbessern - dabei wird besonders auf die Formen der Bilderschließung eingegangen und eine Typologie der vorzufindenden Strategien aufgestellt. In einem zweiten Schritt wird analysiert, welche unterschiedlichen Rollen als soziale Positionierungen in den Audioguides etabliert werden.

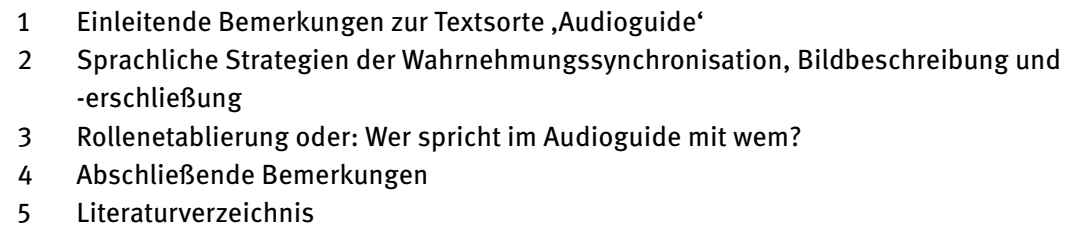

\section{Einleitende Bemerkungen zur Textsorte ,Audioguide}

Wie in anderen Kommunikationsbereichen, die durch funktionale Differenzierung moderner Gesellschaften entstehen, entwickel(te)n sich auch im Bereich der Kunstkommunikation bestimmte Textmuster, um spezifische kommunikative Aufgaben in der sozialen Handlungspraxis zu bewältigen. Solche Textmuster können bestimmten komplexen Handlungen zugeordnet werden und liegen konkreten Textsorten zugrunde. Ein spezifisch ausdifferenzierter Bereich der Kunstkommunikation ist die Ausstellungs- und Museumskommunikation, im Rahmen derer nicht nur Kunst vermittelt wird und Kommunikation über das Kunstwerk stattfindet, sondern die auch einen Ort bietet, an dem die Begegnung mit dem Kunstwerk möglich wird. Eine hierfür spezifisch etablierte Textsorte ist der Audioguide (vgl. dazu auch Fandrych/ Thurmair 2011, 73 ff.).

Audioguides lassen sich in eine Reihe mit anderen Führern (,guides') stellen im Sinne von systematischen Informationssammlungen $\mathrm{zu}$ unterschiedlichen Gebieten (wie Opernführer, Reiseführer, Wanderführer, Restaurantführer etc.), die sich an Nicht-Fachleute richten (zum Vergleich von Audioguides und Reiseführertexten 
vgl. Fandrych/Thurmair 2010). Bevorzugt handelt es sich dabei um stark strukturierte Texte, die zum Nachschlagen bzw. zur selektiven Wahrnehmung geeignet sein sollten. Audioguides sind eine relativ junge Textsorte (sie existieren vermutlich seit gut 50 Jahren; Schulze/Buhl 2012, 29); ursprünglich sind sie als elektronische Museumsführer entstanden, die im akustischen Medium Wissenswertes zu den Objekten im Museum darboten. Mittlerweile gibt es verschiedenste Ausdifferenzierungen von Audioguides, sowohl hinsichtlich des Gegenstands/Themas (Denkmäler, geschichtlich relevante Orte, Stadträume) als auch hinsichtlich der Adressaten (Kinder und Jugendliche, Blinde) und Autoren (z. B. treten in bestimmten Projektzusammenhängen auch Jugendliche als Autoren auf, vgl. die Beiträge in Kunz-Ott 2012). Im folgenden Beitrag geht es spezifisch um Audioguide-Texte als Texte der Kunstkommunikation am Beispiel der Museumskommunikation in Gemäldeausstellungen.

Die Audioguides gehören zu den mündlich realisierten, konzeptuell schriftlichen Textsorten (Gutenberg 2000), d. h. sie werden in der Regel vorformuliert und dann von einem Sprecher / einer Sprecherin mündlich realisiert. Die Rezeption durch den Hörer / die Hörerin findet simultan zur Rezeption des Kunstereignisses statt, die enge Beziehung zwischen Kunstwerk und Text ist konstitutiv für diese Textsorte, genauso wie die Tatsache, dass sich der Hörer unmittelbar vor dem Bild befindet, die Kommunikation also im Museumsraum situiert ist. Es handelt sich also um echte Simultankommunikation im Unterschied zur Vorauskommunikation (z. B. die Lektüre des Ausstellungskatalogs zur Vorbereitung auf den Besuch einer Ausstellung) und zur Anschlusskommunikation (also das Sprechen über ein Kunstereignis nach dessen Rezeption, die Hausendorf (2005) als riskante und somit schwierige Kommunikationspraxis besonders hervorhebt).

Als (gesprochene) Hörtexte unterliegen Audioguide-Texte spezifischen Rezeptionsbedingungen: Sie sind flüchtig (wenn auch durch die technischen Bedingungen der Konservierung vom Rezipienten unbegrenzt oft wiederholbar) und können nur hörend rezipiert werden. Im Unterschied zu anderen Hörtexten, deren Rezeption oft dadurch erschwert wird, dass keine klare Hör-Erwartung besteht, ist diese aber bei den Audioguides durch den gesamten Rezeptionskontext relativ klar konturiert: Sie sind nur in einem spezifischen Kontext, nämlich beim Besuch etwa eines Museums zu rezipieren, man muss sich diese Texte (und die dazugehörigen technischen Geräte) in einem individuellen Akt beschaffen (und oft dafür bezahlen), es gibt Instruktionen für ihre Benutzung. Dieser spezifische situative Rezeptionskontext verbunden mit der internen Struktur der Texte bedingt, dass der Rezipient bzw. die Rezipientin relevante Informationen zu den ausgestellten Kunstwerken erwartet. Unter kunstkommunikativem Aspekt ist diese spezifische Situation auch deshalb interessant, weil sich die für die Kunstkommunikation in anderen Fällen ausgesprochen zentrale Frage „Kunst oder nicht?“ hier erübrigt (was allerdings durch den Kontext ,Museum“ weitgehend auch schon erfolgt). Wenn es einen Audioguide-Text zu einem Objekt gibt, wird diesem der Status ,Kunst“ zugesprochen. Der „Kunstverdacht“ (Hausendorf 2006, 68) hat sich spätestens dann bestätigt. 
Was die mediale Spezifik betrifft, so unterscheiden sich Audioguide-Texte von schriftlichen Kunstbeschreibungen durch die konstitutive Kommunikationssituation ,im Museumsraum vor dem Bild', was in spezifischen, allerdings nicht immer systematisch eingesetzten sprachlichen Strategien deutlich wird (s. dazu Abschnitt 2.1 und Abschnitt 3), und auch in der Nutzung der akustischen Möglichkeiten lassen sich recht unterschiedliche Formen feststellen: Das reicht von nur gelesenen (Informations-)Texten über Einsatz von verschiedenen Klanggestaltungen (Musik, Geräusche) bis hin zu dramaturgisch ausgefeilten Hörszenen.

Audioguide-Texte stellen konstatierend-assertierend auf ein bestimmtes Kunstobjekt orientiertes Wissen bereit; hinzu kommt auch die Intention, durch die Kommunikation über das Kunstwerk die Rezeption von Kunst und die „Kommunikation mit dem Kunstwerk“ zu erleichtern, zu steuern, und vielleicht sogar zu optimieren. Sie sollen das Kunsterlebnis in der sozialen Praxis des Ausstellungs- und Museumsbesuchs verbessern. In diesem Sinne ist die Intention der Audioguides grundsätzlich auch eine didaktische.

Als Texte der Kunstkommunikation können auch Audioguides mindestens die bei Hausendorf $(2005,2006)$ allgemeiner für Kunstkommunikation beschriebenen vier sprachlichen Teilhandlungen mit ihren je spezifischen Funktionen aufweisen: Beschreiben (Was gibt es zu sehen, hören, tasten...?), Deuten (Was steckt dahinter?), Erläutern (Was weiß man darüber?), Bewerten (Was ist davon zu halten?). Hinzu kommt bei Hausendorf 2011 noch das Bezugnehmen (Worum geht es?). In unserem Textkorpus lassen sich diese Teilhandlungen in unterschiedlichem Maße feststellen; dabei könnte man durchaus dem Erläutern einen besonders gewichtigen Stellenwert zuschreiben, da hier am stärksten auf fachgebundenes Wissen referiert wird, das gerade in diesen Texten mit ihrer didaktischen Intention vermittelt werden kann. Das Beschreiben wiederum ist hier verbunden mit einer mehr oder weniger expliziten ,Hörerführung‘, das heißt, die Bildbetrachtung des Hörers / der Hörerin wird geführt, die Aufmerksamkeit geleitet und fokussiert; in dieser Hinsicht weisen die Audioguides latent oder explizit instruktive Anteile auf. Grundsätzlich sind wie auch bei anderen Kunstbeschreibungen (vgl. Klotz in diesem Band) die einzelnen Teilhandlungen sehr häufig ineinander verwoben (Bewertungen etwa klingen immer durch) und bisweilen noch mit anderen sprachlichen Handlungen (z. B. Erzählen) verbunden.

Die Audioguide-Texte zeigen eine klare Struktur: Sie sind in Teiltexte, also Höreinheiten, gegliedert, die sich je auf ein eigenes Kunstwerk beziehen, und durch eindeutige Zuordnung (meist Nummern, die auf die jeweiligen Bilder verweisen) und die technische Gestaltung ist es jedem Benutzer leicht möglich, die Informationen individuell abzurufen. Diese „punktuelle Führung“ wird von den meisten Benutzern gegenüber einer linearen bevorzugt (Schulze/Buhl 2012, 28).

In der einschlägigen Literatur wird seit längerem über die Notwendigkeit von Texten und Informationen für Museumskommunikation diskutiert; mit Dawid/ Schlesinger (2002) gehen auch wir von deren Notwendigkeit aus, eine Position, die durchaus auch durch Forschungen über das Verhalten von Ausstellungsbesuchern 
gestützt wird. Besucher erwarten Texte und sind an zusätzlichen Informationen interessiert; ihr Fehlen wird als irritierend empfunden. Untersuchungen zu schriftlichen Texten zeigen, dass Besucher mehr davon rezipieren als erwartet (vgl. Dawid/Schlesinger 2002, 7 ff.). Der Vorteil von Audioguides gegenüber schriftlichen Museumstexten besteht darin, dass das Hören - im Unterschied zum Lesen - die Bildrezeption nicht beeinträchtigt, also simultan möglich ist, und dass Audiotexte etwas länger sein können als Begleittexte zu Bildern, für die relativ strenge Umfangsbegrenzungen gelten. Der Vorteil von Audioguides gegenüber ,lebenden` Führungen wird in der stärkeren Autonomie des Besuchers gesehen. Als möglichen kommunikativen Nachteil der Audioguides kann man die Tatsache werten, dass im Allgemeinen Kommunikation von Besuchern untereinander weitgehend unterbunden wird.

Die folgenden Ausführungen basieren auf einem Korpus von 50 Texten zu Bildern unterschiedlichster Epochen, die in ganz verschiedenen Museen im deutschsprachigen Raum hängen; damit ist auch sichergestellt, dass die Audioguides von unterschiedlichen Autoren stammen. Die Texte werden schriftlich in orthographisch angepasster Form wiedergegeben.

Im Folgenden sollen mit den Strategien der Bilderschließung und der Rollenetablierung zwei zentrale Aspekte dieser Textsorte untersucht werden.

\section{Sprachliche Strategien der Wahrnehmungssyn- chronisation, Bildbeschreibung und -erschließung}

Die kommunikativen Aufgaben, die sich für Audioguides stellen, sind komplex. Sie sind darauf ausgerichtet, das Kunsterlebnis von Kunstbetrachtern zu verbessern, indem sie auf Kunstobjekte bezogenes Wissen in möglichst gut rezipierbarer Weise präsentieren und dabei die Kunsthaftigkeit der Objekte, die Sinnhaftigkeit und den gehobenen Wert von vertiefter Kunstrezeption im Allgemeinen, und in der jeweiligen Institution im Besonderen, bestätigen. So tragen sie letztlich auch zum Selbstverständnis und zur Selbstvergewisserung bestimmter sozialer Gruppen bei (vgl. Hausendorf/Müller 2015). Audioguide-Texte setzen beim Kunstbetrachter ein Wissen über die Musterhaftigkeit der sozialen Praxis des Ausstellungs-/Museumsbesuchs voraus: Die Betrachter sind mit der typischen räumlichen Organisation von (Gemälde-)Ausstellungen ebenso vertraut wie mit der „Episodenhaftigkeit“ von Ausstellungsbesuchen (vgl. Hausendorf 2014), die an den jeweiligen Exponaten als Kunsteinheiten orientiert ist. Gleichwohl müssen Audioguide-Texte auf ein Hörerwissen und auf vermutete Hörerwissensdefizite ebenso Bezug nehmen, wie sie aus der Fülle der verfügbaren kunstbezogenen Wissensbestände rigoros das auswählen müssen, was sie als für das Kunsterlebnis der Besucher für relevant und interessant, evtl. auch unterhaltsam betrachten. Schon die Auswahl des vermittelten Wissens stellt daher eine Bewertung dar. Dabei scheint die Tendenz zu bestehen, das Wissen und auch die 
Seh- und Deutungsgewohnheiten der im Text modellierten Betrachter als eher niedrig anzusetzen: Der Betrachter wird als ein „etwas dummer“ und „etwas naiver“ Hörer modelliert (vgl. Hausendorf/Müller 2015).

\subsection{Bezugnahme und Synchronisation der Wahrnehmung}

Voraussetzung der Kunstkommunikation mithilfe von Audioguides ist zunächst die Synchronisation der Orientierung zwischen Audioguide-Text und Betrachter. Dabei dient die bereits erwähnte Episodenhaftigkeit des Ausstellungs- bzw. Museumsbesuchs als (stillschweigend als gültig angenommenes) Grundmuster: Als Betrachter widmet man sich als Kunstobjekten identifizierbaren (und entsprechend präsentierten und etikettierten) Gegenständen für einen gewissen Zeitraum intensiver und bewegt sich in einer vorgegebenen oder selbst gewählten Chronologie durch den Ausstellungsraum. Jede intensivere Rezeption eines Kunstgegenstands kann als Episode angesehen werden. Zur Synchronisation der Aufmerksamkeit des Betrachters muss der Audioguide-Text Hinweise auf den Bezug zum jeweiligen Betrachtungsobjekt herstellen: „,[D]as Kunstwerk ist in einer Ausstellung nicht einfach ,da', sondern sein ,Da-Sein' wird demonstriert und präsentiert, in einem emphatischen Sinne gezeigt“ (Hausendorf 2011, 518). Hierfür kann eine Reihe von sprachlichen und nicht-sprachlichen Strategien genutzt werden. Die nicht-sprachlichen Strategien (Nummerierungen, visuelle Symbole etc.) sollen hier nicht weiter behandelt werden. Die sprachliche Bezugnahme kann recht unterschiedlicher Natur sein. Am explizitesten erfolgt sie durch die Nennung von Künstlernamen und Bildtitel zu Anfang des Audio-Textes, wie in Beleg (1):

(1) Barnett Newmans Werk „Mitternachtsblau“ zieht den Betrachter auf magische Weise in seinen Bann. (Barnett Newman, „Mitternachtsblau“)

Dieser explizite Bezug muss nicht textinitial erfolgen, wie Beleg (2) zeigt:

(2) 1918 gründete Kurt Schwitters in Hannover die dadaistische Bewegung „Merz“ [es folgen drei Sätze zur Kunstbewegung „Dada“, CF \& MT]. Ein Zeitungsfetzen mit einer Anzeige der Commerzbank gab seinem Merz-Zyklus den Namen, wozu auch dieses „Merzbild 9B“ gehört. (Kurt Schwitters, „Merzbild 9B“)

Hier wird der Bezug allerdings durch die Nennung des Künstlernamens sowie die Kunstbewegung Merz, die dann im Namen als Bestimmungswort wieder auftritt, auch schon recht explizit hergestellt. Weniger explizit erfolgt die Bezugnahme im nächsten Beleg (3): 
(3) Dieses Bild ist ganz gewiss kein normales Portrait. Und keine Sorge: Die Frau, die der spanische Künstler Pablo Picasso dargestellt hat, hat in Wirklichkeit bestimmt nicht so ausgesehen. (Pablo Picasso, „Frau mit grünem Hut“)

Mit dieses Bild wird dem Betrachter zunächst eine Suchanweisung gegeben, die durch den folgenden Satz allerdings deutlich erleichtert wird, da hier ein wesentlicher Namensbestandteil des Titels (Frau) sowie der Künstlername nachgeliefert werden. Deiktisch vermittelt ist dagegen die Bezugnahme im Beleg (4):

(4) Vor einem abstrakt strukturierten, hellen Hintergrund treten uns hier zwei Männer gegenüber. Sie könnten unterschiedlicher kaum sein. [...] Es sind Vater und Sohn. Der 17-jährige Otto Benesch und sein Vater Heinrich. (Egon Schiele, „Doppelbildnis Heinrich und Otto Benesch“)

Mit hier wird eine Position der Nähe zu einem Kunstwerk evoziert, das vor dem Hintergrund der Episodenhaftigkeit des Ausstellungsbesuchs und dem Muster des Sich-Vertiefens in ein Kunstwerk den Beginn einer solchen Episode markiert. Ansonsten wird die Bezugnahme sprachlich zunächst nur durch die Bildbeschreibung selbst ermöglicht, die allerdings in diesem Fall eine recht eindeutige und einfache Identifikation ermöglichen sollte. Im weiteren Verlauf (im vierten Satz) wird durch die Nennung des Namens der dargestellten Personen dann die Bezugnahme noch weiter vereindeutigt.

Beleg (5) schließlich ist ein Beispiel für einen Audioguide-Text, der den Titel des Bildes an keiner Stelle explizit nennt:

(5) Dieses Bild von Raffael zählt zu den Meisterwerken der Hochrenaissance. Unter Hochrenaissance versteht man eine sehr kurze Zeitspanne [...], in denen die Maler ein Höchstmaß an Ausgewogenheit und Harmonie anstreben. [...] Maria, das Jesuskind und den Johannesknaben [...] die weite, grüne Landschaft [...] idealisierende Darstellung der Madonna [...]. (Raffael, „Madonna im Grünen“)

Die Bezugnahme erfolgt sprachlich durch den Ausdruck „dieses Bild von Raffael“, mit dem eine Suchanweisung gegeben wird, die in der Folge abgeglichen werden muss mit der Bildbeschreibung, in der zwar Bestandteile des Titels indirekt (,Maria“) oder direkt („die weite, grüne Landschaft“; „Madonna“) aufgegriffen werden, aber eben nicht im Wortlaut des Titels.

\subsection{Bildbeschreibung und -erschließung}

Die zentrale Funktion von Audioguide-Texten besteht in der Erschließung und Deutung des Kunstwerkes. Beide Handlungen verbinden sich mit dem Beschreiben und auch mit dem Bewerten des Gemäldes oder bestimmter Aspekte davon (die Verschränkung dieser sprachlichen Handlungen sind für Kunstkommunikation allgemeiner einschlägig, vgl. die Unterscheidung von Ekphrasis und Deskription bei Klotz, in diesem Band). Dabei muss das Kunstwerk (in unserem Fall: das Gemälde), das in 
einem visuell-statischen, zweidimensionalen Medium ,stumm' in einer entsprechenden Institution präsent ist und präsentiert wird, linearisiert beschrieben und gedeutet werden. Konkret müssen die Simultanität und Vielgestaltigkeit des Bildes sowohl textuell als auch visuell linearisiert werden: Man muss auch bei der visuellen Wahrnehmung bestimmte Dinge zuerst fokussieren, sich für eine Betrachtung der ,Totalen“ oder bestimmter Details entscheiden und die visuelle Perzeption insgesamt so in eine Reihenfolge bringen. Der Text schafft also einen fiktiven ,Bildraum` bzw. eine fiktive ,Bildsituation', welche die Betrachter/innen häufig miteinschließen (vgl. etwa Beleg 4). Dabei werden die Belebung des Bildgeschehens bzw. die Führung durch Raum oder Situation meist unmittelbar und explizit mit der Deutung verknüpft. Die Wahl des Einstiegs und die Auswahl der sukzessive besprochenen Aspekte durch die Textautoren etablieren bestimmte Bildausschnitte als relevante, wichtige, für das Verstehen und die Interpretation bedeutungsvolle Bildelemente. Häufig wird diese Sinnaufladung auch an der Textoberfläche verstärkt und bestätigt.

Es lassen sich nun eine Reihe von sprachlich-textuellen Verfahren unterscheiden, wie der Zugang zum Bild und zur Bilddeutung gestaltet werden kann. Generell kann man feststellen, dass in unserem Korpus mehrheitlich versucht wird, Ambiguitäten aufzulösen und über verschiedene Wege als ,gültig‘ gesetzte Interpretationen und Deutungen herzustellen und dem Betrachter nahezulegen. Einige dieser Strategien wollen wir im Folgenden etwas näher anhand von Belegen besprechen.

\subsubsection{Dynamisiertes Standbild und historisch-biographische Identifikation}

(6) [Sprecher] Vor einem abstrakt strukturierten, hellen Hintergrund treten uns hier zwei Männer gegenüber. Sie könnten unterschiedlicher kaum sein: Jung, hager und blass der eine, untersetzt und mit rötlicher Gesichtsfarbe der andere. Es sind Vater und Sohn. Der 17-jährige Otto Benesch und sein Vater Heinrich. Der Maler Egon Schiele kannte sie gut, denn Bahninspektor Heinrich Benesch war einer seiner ersten und treuesten Förderer. [...] Der Maler hat die beiden in ein spannungsreiches Verhältnis gesetzt. Dazu die Direktorin des Lentos Stella Rollig:

[O-Ton Stella Rollig]

Egon Schieles Doppelbildnis Heinrich und Otto Benesch hat mich immer fasziniert, weil es ein so ausdrucksstarkes Portrait von zwei Männertypen ist. Außergewöhnlich ist die Haltung der Männer. Der Sohn, ein gutaussehender, feingliedriger, junger Mann, wird von seinem streng blickenden Vater abgeschirmt von Kontakt und zwar von Kontakt ganz direkt mit den Betrachtern vor dem Gemälde. Der Sohn wäre eigentlich in der Position mit uns vor dem Bild in einen Dialog zu treten, doch der Vater wendet uns ganz ausdrücklich den Rücken zu und ist im Begriff, auch den Sohn wegzudrehen oder zu verhindern näher zu kommen. (Egon Schiele, „Doppelbildnis Heinrich und Otto Benesch“)

Dieser Audio-Text mit seinen beiden Sprecher-Rollen (vgl. dazu Abschnitt 3) setzt sofort bei einer deutenden Charakterisierung der beiden im Bild dargestellten Figuren ein („könnten unterschiedlicher kaum sein“). Von Beginn an wird die Beschreibung des Standbildes mit Handlungspotenzial und Situationspotenzial aufgeladen, die 
scheinbar vom Bild selbst ausgehen („treten uns hier zwei Männer gegenüber“; ,wird abgeschirmt von Kontakt“; , ist im Begriff, auch den Sohn wegzudrehen“; Popp 2013, 136 ff. spricht hier von „aktivierten Bildern“). Diese Dynamisierung bettet das Bildgeschehen in eine angenommene Handlungsverkettung ein, die dann als Grundlage zur weiteren Interpretation dient. Präsupponiert wird durch die vom Text geschaffene fiktive Handlungskonstellation auch die Position des Betrachters in einer Näheposition zu den auf dem Gemälde dargestellten Figuren: Sie scheint die spontane Aufnahme eines Gesprächs durch die Figuren im Bild zu ermöglichen (dass diese beabsichtigt wird, legt der Text ja nahe). Gleichzeitig besteht aber noch eine gewisse Distanz, die eine Intervention des Vaters ermöglicht.

Darüber hinaus wird hier besonders stark mit der Strategie der biographisch-historischen Deutung gearbeitet, die durch den Bildtitel natürlich naheliegt. Dennoch ist auffallend, wie ausschließlich der Text mithilfe von zusätzlichen erläuternden Fakten eine biographische Deutung nahelegt und letztlich auch in dieser Deutung verharrt. Hierzu wird am Ende des Audio-Textes sogar noch ein Zusatztext angeboten, der bei Interesse abgerufen werden kann: ein Auszug eines Briefes von Heinrich Benesch an Egon Schiele „kurz vor der Entstehung dieses Gemäldes“. Die Interpretation läuft letztlich auf die Frage hinaus, was für ein Vorfall sich zwischen Schiele und Benesch ereignet haben könnte, der Schiele dazu veranlasste, Heinrich Benesch so wenig sympathisch darzustellen.

Daneben arbeitet der Audiotext mit der fiktiven Aufhebung der Grenze zwischen Bild und Betrachter: Der Sohn wird vom Vater ,,abgeschirmt“, „und zwar von Kontakt ganz direkt mit den Betrachtern vor dem Gemälde“; der Sohn wäre „eigentlich in der Position mit uns vor dem Bild in einen Dialog zu treten, doch der Vater wendet uns ganz ausdrücklich den Rücken zu“. Die Wirkung eines solchen textuellen Verfahrens kann vielfältig sein: Auf einer ersten Ebene wird damit zunächst der Betrachter aus seiner eher statisch-rezeptiven Rolle fiktiv in eine dynamische Situation versetzt und somit „aktiviert“. Gleichzeitig suggeriert die Distanzaufhebung, dass die Bilddeutung durch eine solche Situationseinbindung und scheinbare Unmittelbarkeit des Erlebens auf besonders relevante und treffende Art zu erfassen sei. Im hier vorliegenden Fall wird das Verhalten der Personen einem Betrachter gegenüber (Schiele / dem Museumsbesucher) zur Grundlage von psychologischer Interpretation (so heißt es auch im weiteren Textverlauf „Die Körpersprache wird zum Ausdrucksmittel seelischer Zustände“). Verkürzt gesagt, nehmen wir das Bild als Abbild einer vom Künstler wahrgenommenen Realität und Realitätsdeutung wahr, als symbolhaft verdichtetes Psychogramm von historischen Personen.

\subsubsection{Orientierung im (vom Bild gestifteten) Raum}

Eine weitere Strategie besteht in der engen Orientierung an der vom Bild ,vorgegebenen' räumlichen Strukturierung. Auch diese Vorgehensweise dient der Stiftung 
bzw. Herstellung von „Wiedererkennen“ beim Hörer/Betrachter (vgl. Gehlen 1965 und Popp 2013, 33 ff.), nicht (in erster Linie) dem Infragestellen. Ein gutes Beispiel findet sich beim Audio-Kommentar zu Bruegels Bild „Die Jäger im Schnee“:

(7) Dieses letzte Bild aus dem Jahreszeitenzyklus von Pieter Bruegel ist zugleich das bekannteste und populärste der ganzen Serie. [...] Der grün-blaue Himmel lässt die frostige Kälte nachempfinden, in der die Welt erstarrt ist. Ganz vorne drückt der schwere Schnee die dürren Ranken eines kahlen Brombeerstrauchs nieder. Müde stapfen die Jäger und ihre Hunde mit magerer Beute dem tieferliegenden Dorf entgegen. Die Jäger kehren uns den Rücken zu. Gemeinsam mit der Baumreihe lenken sie unseren Blick hinab in die ferne Tiefe vorbei an den ameisengroßen Schlittschuhläufern bis zum eisbedeckten Gebirge am Horizont. Wieder sind die eigentlichen jahreszeitlich typischen Tätigkeiten an den Rand gedrängt. Ganz links vor dem Wirtshaus entfachen die Menschen ein Feuer, um ein geschlachtetes Schwein zu sengen. (Pieter Bruegel, „Die Jäger im Schnee“; Hervorhebungen hier und im Folgenden CF \& MT)

Es wird vom Gesamteindruck zu den Details vorangeschritten, wobei die Aufmerksamkeit vom Vordergrund und Zentrum des Bildes in den „Hintergrund“ und an die Peripherie geleitet wird: „ganz vorne“; „dem tieferliegenden Dorf entgegen“; „lenken [...] unseren Blick hinab in die ferne Tiefe“; „sind [...] an den Rand gedrängt“. Dies wird nicht zuletzt durch die „Animierung“ der dargestellten Gegenstände und Personen geleistet: vom grün-blauen Himmel über den schweren Schnee bis hin zu den Jägern und der Baumreihe gehen Handlungen aus, die uns als Betrachter lenken bzw. im Bild Dynamik erzeugen. Die Identifizierung erfolgt mithilfe von deiktisch aufzufassenden Definitartikeln, die sich wie Suchanweisungen lesen (,die Landschaft“; „der grün-blaue Himmel“; „der schwere Schnee“; „die Jäger“; „dem tieferliegenden Dorf entgegen“; „mit der Baumreihe“; „,in die ferne Tiefe vorbei an den ameisengroßen Schlittschuhläufern“). Auch hier erfolgt die Deutung und Wertung wesentlich durch die attributiv bzw. adverbial verwendeten Adjektive, die sich so mit der räumlichen Erschließung des Bildes eng verbinden (,frostige Kälte“; „schwerer Schnee“; „dürre Ranken“, „kahler Brombeerstrauch“; „müde stapfen die Jäger“; „mit magerer Beute“ ...). So verbindet sich hier eine am Raum orientierte, stark perspektivierende Bildbeschreibung mit dem Aufrufen von Objekten, Personen und Landschaftsaspekten, die „wiedererkannt“ werden, und einer v. a. in Attribuierungen eingelagerten Deutung.

\subsubsection{Bild als Angebot zur fiktiven Teilhabe (,Lebendigmachen einer Szene`)}

Ein weiteres textuelles Verfahren der Bilderschließung besteht darin, das Bild ,zum Leben' zu erwecken. Ein fiktiver Sprecher nimmt eine Perspektive ,aus dem Bild‘ an und spricht den Rezipienten direkt an - es wird ein Dialog inszeniert, der den Hörer mit Handlungsaufforderungen und -alternativen konfrontiert und ihm die Möglichkeit der echten Partizipation an der im Bild dargestellten Situation anbietet (,Kommen Sie doch näher, setzen Sie sich nieder auf ein Glas Bier! Oder wollen Sie noch ein wenig 
stehenbleiben und die Leute betrachten? Was sehen Sie denn? Ja, die Kinder hier vorne sind ganz vertieft; die beiden rechts spielen im Sand [...]“; vgl. ausführlicher Abschnitt 3.1.3). Die Grenze zwischen Bild und Betrachter wird hier also scheinbar in umgekehrter Richtung aufgehoben als bei 2.2.1; durch die fiktive Teilnahme des Betrachters an einer im Bild dargestellten Situation soll die Beschreibung des Dargestellten und auch schon die Deutung intensiver, direkter, persönlicher erfahren und nachvollzogen werden können. Wie bei Beleg (6) wird dabei präsupponiert, dass sich der Betrachter bzw. die Betrachterin in einer Näheposition befinde, die ihm/ihr eine Teilnahme unmittelbar ermöglicht, die allerdings doch noch eine gewisse Distanz zum dargestellten Geschehen aufweist: Die Stimme aus dem Bild heraus kann problemlos mit uns kommunizieren, befindet sich also in Hör- und Sprechweite, lädt aber doch zum Nähertreten ein.

\subsubsection{Bilder als ,Zeugen` gesellschaftlicher und historischer Umstände}

Ein weiteres textuelles Verfahren der Annäherung an die Bilderschließung und -deutung besteht darin, sie als Zeugen einer historisch-sozialen Realität zu lesen. Das Bild gewinnt eine Art ,dokumentarischen' Charakter, wird in einen erläuternden historisch-narrativen Diskurs eingewoben. So wird es nicht (mehr) in erster Linie als Kunstwerk thematisiert, sondern als Zeitzeugnis. Ähnlich wie bei der biographischhistorischen Identifikation (2.2.1) erfolgt dadurch eine ,Vereindeutigung der Bildaussage, der Kunstkommunikation, die scheinbar vom Bild selbst ausgeht; so wird das Bild in seiner Kunsthaftigkeit und Mehrdeutigkeit teils kaum mehr thematisiert. Gleichzeitig wird es als eine Art Abbild einer ,objektiven' historischen Realität konzeptualisiert, die anhand des Dargestellten rekonstruiert und bestätigt werden kann (was durch weitere Sprecherrollen unterstützt wird), vgl.:

(8) [...] Doch womit sind eigentlich die Leute auf diesem Gemälde beschäftigt? [...] Gebückt und auf Knien klauben sie aus dem Flussbett der Isar Kalksteinbrocken, die anschließend in Fuhrwerken abtransportiert und in nahegelegenen Kalköfen gebrannt werden. Ein Zeitgenosse berichtete:

[zweiter Sprecher]

Es macht einen eigenen Eindruck auf den grellweißen Kiesbänken des Stromes diese weißen Gestalten gleich mittäglichen Gespenstern einherwandeln zu sehen, wie sie sich nach den Steinen bücken.

[Sprecherin]

Die Hosen trugen die Kalksteinsammlerinnen bei der Arbeit zum Schutz über ihren Röcken. Gebrannten Kalk brauchte man zur Herstellung von Mörtel und der wurde damals, in der zweiten Hälfte des 19. Jahrhunderts, in großen Mengen zum Häuserbau in den Städten benötigt. (Joseph Wenglein, „Kalksteinsammlerinnen im Isarbett“) 


\subsubsection{Das Bild als Verkörperung eines künstlerischen Konzepts}

Eine stärker kunsthistorisch-didaktisierend orientierte Beschreibung und Deutung von Kunstwerken erfolgt über die Fokussierung der für eine ,Schule“ typischen darstellerischen/malerischen Eigenschaften. Hierbei handelt es sich in gewisser Weise um das Gegenstück zum situativen oder topographischen „Wiedererkennen“: Situation und Räumlichkeit rücken in den Hintergrund, darstellerische Details in ihrer ,Typik‘ rücken in den Vordergrund des Audio-Textes und werden mit einem kunsthistorischen Exkurs verbunden (einer „Erläuterung“, vgl. Hausendorf 2011). Diese Strategie wird v. a. auch bei moderner Kunst angewandt (vgl. Gehlen 1965; Popp 2013, 33 ff., die auch die Debatte um die existenzielle „Kommentarbedürftigkeit“ abstrakter Kunst bzw. die autonome, andersartige Kommunikationsfähigkeit von Kunstwerken [nach Luhmann 1997] zusammenfasst). Allerdings beschränkt sie sich nicht darauf, vgl. das folgende Beispiel von Raffael:

(9) Dieses Bild von Raffael zählt zu den Meisterwerken der Hochrenaissance. Unter Hochrenaissance versteht man eine sehr kurze Zeitspanne - in etwa die Jahre von 1500 bis 1520, in denen die Maler ein Höchstmaß an Ausgewogenheit und Harmonie anstreben. Raffael gestaltet dieses Bild als streng geometrische Komposition. Er fasst Maria, das Jesuskind und den Johannesknaben zu einem Dreiecksbild zusammen. Ein weiteres Dreieck bilden Jesus und der Kreuzstab des Johannes. Auch im Hintergrund links wiederholt sich das Dreieck in Form eines Hügels. Das heißt, Raffael spielt bis ins Detail mit geometrischen Entsprechungen. Trotz dieses strengen Aufbaus wirkt das Bild nicht starr, sondern natürlich und lebendig, denn die drei Personen sind durch Blicke und Berührungen miteinander verbunden. (Raffael, „Madonna im Grünen“)

Hier dient die Bildbeschreibung ganz der Etablierung und Bestätigung der Repräsentativität des Bildes für eine bestimmte Kunstrichtung, in diesem Fall der Hochrenaissance. Diese wird als von einem „Höchstmaß an Ausgewogenheit und Harmonie“ geprägt charakterisiert, was sich in der „streng geometrische[n] Komposition“ niederschlägt. Dieses Kompositionsprinzip wird dann an verschiedenen Bildausschnitten aufgezeigt und bestätigt: „[...] fasst [...] zu einem Dreiecksbild zusammen“; „Ein weiteres Dreieck bilden [...]“; , ,auch im Hintergrund links wiederholt sich das Dreieck“. Im Anschluss wird die sich daraus ergebende Deutung nochmals explizit konstatiert („Das heißt, Raffael spielt bis ins Detail mit geometrischen Formen“).

Auch bei abstrakter Kunst wird der Fokus häufig zum einen auf das künstlerische Programm, zum anderen auf die Mal- und Farbtechnik gelenkt, die für dieses künstlerische Programm stehen. Auch dadurch werden Bilder zu Symbolen für Kunstrichtungen, allerdings ist für wenig mit abstrakter Kunst vertraute Betrachter der Kunstkommentar vielleicht noch essentieller für die Kunstrezeption, vgl.:

(10) Barnett Newmans Werk „Mitternachtsblau“ zieht den Betrachter auf magische Weise in seinen Bann. Man fühlt sich von einem unermesslichen Farbraum umhüllt, dessen Ausdehnung, Dichte und Tiefe durch den Kontrast mit dem weißen Band links und dem türkisblauen Streifen rechts noch verstärkt wird. Barnett Newman entwickelte zusammen mit seinen amerikanischen 
Künstlerkollegen Jackson Pollock, Mark Rothko und Clyfford Still den Abstrakten Expressionismus. Newmans Malerei erschließt sich durch seine kunsttheoretischen Schriften. [...] Seine Malerei sollte dem Betrachter die Gelegenheit geben, sich aus dem festen Gefüge seiner Umgebung zu lösen und uneingeschränkte Freiheit zu erleben. Dazu konfrontiert er uns mit einer riesigen monochromen Fläche, die keinerlei Anhaltspunkte für vertraute Vorstellungen bietet. Stattdessen erfahren wir uns beim Anblick des Bildes selbst als Betrachtenden und werden uns des eigenen Ichs bewusst. (Barnett Newman, „Mitternachtsblau“)

Nach einer (suggestiv-emotionalen) Schilderung eines Gesamteindrucks bzw. -gefühls, das die Malerei „dem Betrachter“ vermittelt, setzt der Text bei der kunsthistorischen Einordnung und der Erläuterung des kunsthistorischen Programms des Malers an, aus dem eine angemessene Rezeptionshaltung und auch die Sinnhaftigkeit abgeleitet werden. Es wird sozusagen eine theoretische Folie erarbeitet, auf welcher dann die visuelle Darstellung interpretiert wird.

\section{Rollenetablierung oder: Wer spricht im Audio- guide mit wem?}

Audioguides dienen dazu, eine bestimmte soziale Praxis im Bereich der Kunstkommunikation zu vollziehen. Sie leisten dies unter den spezifischen Bedingungen der unmittelbaren Kommunikation vor dem Kunstwerk. Dazu etablieren sie verschiedene Rollen, die mit sozialen Positionierungen verbunden sind. Dabei sind die Rollen auf Seiten der Sprechenden wesentlich vielfältiger als auf Seiten der Hörenden.

\subsection{Wer spricht?}

Hörtexte in den Audioguides ermöglichen durch Stimmwechsel und damit unterschiedliche Stimmen problemlos die Einbeziehung verschiedener Sprecherinstanzen. Dabei ist in der Regel ein Sprecherwechsel auch inhaltlich motiviert. Es gibt zwei Sprecherrollen, die explizit und namentlich angekündigt werden, nämlich ausgewiesene Experten (meist Museumsdirektoren bzw. -referenten oder Ausstellungskuratoren) und den Künstler/die Künstlerin selbst. Andere Sprecherrollen müssen sich auf andere Weise erschließen (z. B. bei Sprechern ,aus dem Bild heraus‘ auch durch entsprechende Geräusche, eine spezifische Sprechweise).

\subsubsection{Der kompetente Betrachter}

Die häufigste Sprecherrolle ist ein Sprecher, der ebenfalls das Bild betrachtet, der aber einen deutlichen Wissensvorsprung hat. Dieser kann sich auf das ,richtige Sehen“ 
beziehen, er kann aber auch Kontextwissen verschiedenster Art betreffen. Wir haben es also bei dieser Sprecherrolle mit einem kompetenten Betrachter zu tun. Die Rolle als Betrachter zeigt sich sprachlich an verschiedenen Strategien, besonders deutlich durch die Verwendung des Pronomens wir, wenn der Sprecher dadurch den Betrachter mit einbezieht; wie in (7) oben (,Die Jäger kehren uns den Rücken zu. Gemeinsam mit der Baumreihe lenken sie unseren Blick hinab in die ferne Tiefe“) oder in (4) oben (,... treten uns hier zwei Männer gegenüber“), bei dem stärker noch die gemeinsame Position betont wird, und noch deutlicher in (11), bei dem betrachtender Sprecher und betrachtender Hörer gemeinsam in das Bild eintauchen:

(11) Es [das Bild, CF \& MT] zeigt die Dorfstraße des kleinen bayerischen Örtchens Aresing in hellem Sonnenschein. Der Schlagschatten des uns entgegenkommenden Bauern lässt darauf schließen, dass Mittagszeit ist. Von Aresing sind nur einige wenige Häuser zu sehen, die Straße verläuft in die freie Landschaft, wir befinden uns wohl am Dorfausgang. (Franz von Lenbach, „Dorfstraße von Aresing“)

Distanzierter zeigt sich diese kompetente Sprecherrolle in der Verwendung des generalisierenden Pronomens man, das die Hörerrolle und auch andere Referenzrollen umfassen kann (z. B. „man erkennt kaum“ oder „im Hintergrund sieht man“). Durch die Verwendung des generalisierenden Personalpronomens werden die Aussagen stärker legitimiert. In dieser Rolle erscheint in unseren Daten niemals das deiktische Pronomen ich.

Dass dieser ebenfalls das Bild betrachtende Sprecher einen Wissensvorsprung hat, insofern also ein kompetenter Betrachter ist, wird an ganz unterschiedlichen sprachlichen Erscheinungen deutlich. Zum einen ,weiß' dieser Sprecher, wie man das Bild ,richtig‘ betrachtet: Das kann sich auf räumliche Hinweise beziehen (wie in (12)/ (13)) oder - sehr häufig - modal gemeint sein (wie in (14)/(15)):

(12) Am besten wirkt das Bild, wenn Sie ein paar Schritte zurücktreten. Die vielen kleinen Farbtupfen verbinden sich dann in Ihrem Auge zu einer Gartenlandschaft mit Bäumen, Büschen und einem Meer aus Blumen (Curt Hermann, „Im Garten von Schloß Pretzfeld“, zitiert bei Popp 2013, 120)

(13) Das Entstehungsdatum des Bildes finden Sie im Gewandausschnitt der Madonna. (Raffael, „Madonna im Grünen“)

(14) Schauen Sie sich einmal die Arme des Vaters näher an: Er hatte ursprünglich beide Hände in den Jackentaschen vergraben, erst nachträglich ... (Egon Schiele, „Doppelbildnis Heinrich und Otto Benesch“)

(15) Und wenn Sie sich den durchsichtig zarten Stoff des Kleides noch etwas genauer anschauen, werden Sie feststellen, dass er überhaupt nicht plastisch wirkt. (Gustav Klimt, „Margarete Stoneborough-Wittgenstein“) 
Was hier funktional durch den Sprecher geleistet wird, ist, das Beschreiben dessen, was man sieht, zu unterstützen - entweder durch konkrete räumliche Hinweise oder durch modale Hinweise. Dabei finden sich - mehr oder weniger abgeschwächte Aufforderungen, bestimmte Handlungen zu vollziehen. Hier wird die mediale Spezifik der Audioguides gegenüber schriftlichen Texten besonders deutlich: in Beleg (12) wird (was allerdings sehr selten ist) explizit auf die räumlichen Bedingungen der Bildbetrachtung Bezug genommen, in (15) auf die Möglichkeiten der sukzessiven Bildbetrachtung, die mit räumlichen Positionsveränderungen verbunden sein können (,noch etwas genauer anschauen“).

Grundsätzlich ist ja das Beschreiben, wie es in den Audioguides passiert, insofern problematisch, als es nicht wie etwa eine Bildbeschreibung in literarischen Texten erfolgt, um dem Leser ein Bild durch die Übersetzung in Sprache überhaupt anschaulich zu machen und zu vermitteln, sondern es handelt sich bei den Audio-Führungen um eine Beschreibung angesichts dessen, dass der Rezipient das Beschriebene auch vor sich hat, also gleichzeitig mit der Beschreibung (als der verbal vermittelten akustischen Wahrnehmungserfahrung) selbst visuell den Akt der Wahrnehmung erfährt. Eine derartige Beschreibung ist in unseren Augen dann funktional, wenn das Sehen geschult bzw. unterstützt werden soll, indem etwa der Hörer beim Vorgang des Betrachtens ,geführt‘ wird (wie es in diesem spezifischen Fall der Museumskommunikation auch erwartet wird), und wenn - was beim Beschreiben in aller Regel mitschwingt - die Beschreibung ohnehin mit anderen sprachlichen Handlungen verwoben ist und insofern über das reine Beschreiben dessen, was objektiv zu sehen ist, hinausgeht, indem z. B. Erläuterungen und Deutungen angeboten werden, Gewichtungen vorgenommen werden, zeitliche Dimensionen thematisiert werden und anderes mehr.

Der kompetente Sprecher hat aber zum anderen sehr viel Kontextwissen und Fachwissen der unterschiedlichsten Art, das er im Audioguide zur Sprache bringt; angeführt werden Informationen zur Bildkomposition, Licht und Farbgestaltung, technische Details, aber auch weitergehende Kontextinformationen kunstgeschichtlicher Art zur Entstehungsgeschichte, Position im Werk / in der Kunstgeschichte etc. Dann kommt zum Beschreiben das Deuten, das Erläutern oder auch das Bewerten hinzu (s. dazu die entsprechenden Belege im Abschnitt 2.2). Die häufigste Sprecherrolle in den Audioguides ist also Betrachter und fachliche Autorität in einem.

\subsubsection{Der Kunstexperte}

Eine weitere Sprecherrolle, die häufig auftritt, ist die des Kunstexperten: Museumsreferenten, Ausstellungskuratoren etc. Diese werden - explizit eingeführt und namentlich identifiziert - oft als authentische Sprecher im O-Ton herangezogen - dann auch konzeptionell meist deutlich mündlicher. Sie geben entweder weitere Informationen, erläutern also (wie in (16) die Entstehungsgeschichte) oder aber sie bringen ihre sub- 
jektive Sicht des Bildes, ihre persönliche Meinung zum Ausdruck, werten also (wie in (17)), manchmal auch beides.

(16) Der Anlass für das Bild war: 1756 wurde die enge Liaison mit dem König aufgehoben und sie wurde wieder in die Kirche aufgenommen, der König auch. Und sie konnte zu Beginn des Jahres im Februar der Königin als Hofdame vorgestellt werden, weil sie nicht mehr die Mätresse war, aber noch die Freundin und Beraterin. Und als Freundin und Beraterin erscheint sie eben in diesem Bild. (François Boucher, „Bildnis der Marquise de Pompadour“)

(17) Egon Schieles Doppelbildnis Heinrich und Otto Benesch hat mich immer fasziniert, weil [...]. [...] und ich spekuliere manchmal darüber, welcher Vorfall diesem Gemälde vorhergegangen ist. (Egon Schiele, „Doppelbildnis ...“)

Gerade die im O-Ton deutlich mündlicher realisierte subjektive, oft auch emotionale Bewertung des Bildes soll vielleicht einen anderen, emotionalisierteren Zugang zum Kunstgenuss ermöglichen und dem Hörer erleichtern, ähnliche Ansichten und Bewertungen zu entwickeln.

Häufig besteht die Wertung bei den Experten wie auch bei den kompetentbetrachtenden Sprechern auch darin, die Kunsthaftigkeit und Relevanz des Werkes zu bestätigen. So wird im Audiokommentar zu Leonardo da Vincis „Maria mit dem Kinde“ bereits am Anfang die kunstgeschichtliche und künstlerische Relevanz bzw. der Wert des Malers und seines Werkes (trotz des jugendlichen Alters bei der Entstehung) konstatiert (,große Meisterschaft [...] zeigt sich schon“) und auch gegen offensichtliche Schwächen („nicht immer zum Wohle der Werke“; „leicht gekräuselt“; „misslang die Mischung des Bindungsmittels“), die dem nicht kompetenten Betrachter wahrscheinlich gar nicht aufgefallen wären, argumentativ verteidigt:

(18) [...] Leonardo war erst 21 Jahre alt, als er 1473 Maria mit dem Kinde schuf. Doch seine große Meisterschaft, die ihn zu einem der bedeutendsten Maler der Welt werden ließ, zeigt sich schon an diesem Gemälde. [...] Dieser neuartige Sfumato genannte Effekt verdankt sich Leonardos verfeinerten Ausdrucksmitteln. [...] Dass seine technische Experimentierfreude nicht immer zum Wohle der Werke wirkte, weiß man spätestens seit dem Abendmahl. Hier zeigt sich dies am Antlitz der Maria. Es ist, wenn Sie genau hinsehen, leicht gekräuselt. Wahrscheinlich misslang Leonardo die Mischung des Bindemittels. Jugendliche Unerfahrenheit? Auch inhaltlich zeigt sich, dass Leonardo noch ein junger Maler war. (Leonardo Da Vinci, „Maria mit dem Kinde“)

\subsubsection{Der Sprecher aus dem Bild heraus}

Schließlich gibt es eine weitere, eher selten eingeführte Sprecherrolle; das ist ein Sprecher gleichsam aus dem Bild heraus. Die Intention dieser Sprecher ist, auf die eine oder andere Weise ein Eintauchen in das auf dem Bild Dargestellte zu ermöglichen. Häufig ist dies verbunden mit einer passenden Geräuschkulisse. In diesem Fall werden die Möglichkeiten, die der Audioguide als akustisches Medium bietet und 
die ihn grundlegend von schriftlichen Texten unterscheiden, adäquat und funktional genutzt.

(19) Kommen Sie doch näher, setzen Sie sich nieder auf ein Glas Bier! Oder wollen Sie noch ein wenig stehenbleiben und die Leute betrachten? Was sehen Sie denn? Ja, die Kinder hier vorne sind ganz vertieft; die beiden rechts spielen im Sand, ganz unter der Obhut ihrer Mutter mit grünem Sonnenschirm. Das Kind in rotem Kleid läßt sich etwas zu trinken geben, wohlig schließt es dabei die Augen. Beobachtet der Mann links neben dem Baum die kleine Szene? Auf jeden Fall ist er, anders als das trinkende Mädchen und seine Betreuerin, wohlhabend, so wie überhaupt in diesem Münchner Biergarten ganz unterschiedliche sozialen Schichten vertreten sind. Sie alle vereint das eine: Das Bier, das als verbindendes Thema nicht unauffällig braun von Max Liebermann gemalt wurde, sondern in einer Mischung aus Dunkelviolett und Rot. So scheint es als Sprengsel auch weiter hinten im Bild auf. (Max Liebermann, „Münchner Biergarten“)

Hier führt der Sprecher den Betrachter auch wieder aus dem Bild heraus und übernimmt - ohne großen Bruch - wieder die Rolle des kompetenten Betrachters.

In diesen Fällen entsteht durch den Audioguide unmittelbar eine Kommunikation mit dem Kunstwerk, indem die Möglichkeit geschaffen wird, sich direkt in die abgebildete Szene hineinzuversetzen.

\subsubsection{Der Künstler}

Audioguides bieten eine gute Möglichkeit, Originalquellen und Zitate einzuführen (besser als sogenannte Museumstexte, also schriftliche Begleittexte). Häufig kommen damit Künstler selbst zu Wort, die sich zu ihrem Bild äußern, zu Entstehungsbedingungen, Absichten oder anderen Umständen u. ä. So z. B. Kokoschka in der programmatischen Äußerung zu seinem Bild „Linzer Landschaft“: „So was Fades hab ich noch nie gesehen, aber ich werde was draus machen“ (zitiert im Audioguide) oder Karl Hagemeister, der (vgl. (20)) seinen Schaffensprozess reflektiert. Kurt Schwitters wiederum (vgl. (21)) bietet neben einem Verweis auf die Entstehung seines Werkes auch eine Interpretation, die das Verständnis des Bildes ganz wesentlich erhöht.

(20) Hier konnte ich den Kampf der Elemente fühlen; die Allmacht in der Weite des Meeres; den Kampf der alten Buchen mit Wind und Wetter, und das kosmische Leben offenbarte sich mir ganz. (Karl Hagemeister, „Bäume am Seeufer - Ostseeküste“, zitiert bei Popp 2013, 122)

(21) Man kann auch mit Müllabfällen schreien, und das tat ich, indem ich sie zusammenleimte und -nagelte. Ich nannte es „Merz“, aber es war mein Gebet über den siegreichen Ausgang des Krieges - denn noch einmal hatte der Frieden gesiegt. Kaputt war sowieso alles und es galt, aus den Scherben Neues zu bauen. Das aber ist „Merz“: Ich malte, nagelte, klebte, dichtete. (Kurt Schwitters, „Merzbild 9B“)

Die Sprecherrolle des Künstlers wird also, wie die eben angeführten Belege zeigen, in unterschiedlichen Funktionen eingesetzt, manchmal soll sie das Verständnis für das 
Bild erhöhen, manchmal eher ein Eintauchen in den Schaffensprozess ermöglichen und damit dem Betrachter vielleicht wiederum eine intensivere Kommunikation mit dem Kunstwerk ermöglichen.

\subsubsection{Der Techniker}

Ganz selten gibt es in den Audioguides Metakommentare, vor allem technische Informationen (,Mit der gelben Taste rechts oben am Gerät können Sie Objekte ihrer Wahl speichern...“). In diesem Fall spricht ein Sprecher, der nicht notwendigerweise auch Betrachter des Bildes ist. Metakommentare können auch - das hängt von der Textstruktur der Audioguides ab - auf zusätzliche Informationen verweisen, die in einer hierarchisch niedrigeren Textebene zur Verfügung stehen (zu Texthierarchien siehe Dawid/Schlesinger 2002, 35 ff.), z. B.:

(22) Interessant ist auch ein Brief, den Heinrich Benesch kurz vor der Entstehung dieses Gemäldes an Egon Schiele schrieb. Wenn Sie eine Passage daraus hören möchten, drücken Sie jetzt bitte die grüne Taste. (Egon Schiele, „Doppelbildnis ...“)

\subsection{Mit wem wird in den Audioguides gesprochen?}

Wer einen Audioguide rezipiert, ist neben seiner Rolle als hörender Adressat der Texte gleichzeitig auch Besucher des jeweiligen Museums bzw. der Ausstellung und gleichzeitig Betrachter der ausgestellten Werke und als solcher kunstinteressiert. Dies ist die zentrale Rolle, die in den Audioguides konturiert wird. In ein- und ausleitenden Teilen kann auch die Rolle als Museumsbesucher etabliert werden. Hausendorf (2014) sieht hier eine Rolle als Nutznießer, erkennbar an Äußerungen wie „präsentieren wir Ihnen“ oder „wünschen wir Ihnen einen erlebnisreichen Rundgang“ u. ä. Übergreifend und durchgehend wird der Hörer aber als Betrachter positioniert, als ein kunstinteressierter Laie: für ihn wird beschrieben, gedeutet, erläutert und bewertet. Dabei soll ihm die Rezeption von Kunst und die Kommunikation mit dem Kunstwerk ermöglicht und erleichtert und letztlich optimiert werden.

Dazu dienen zum einen Bildbeschreibungen, die es dem betrachtenden Hörer ermöglichen, assoziativ in das Geschehen einzutauchen. Dies geschieht mit expliziten Aufforderungen und/oder entsprechender akustischer Untermalung (wie oben in (19)). Dies geschieht aber auch durch wertende Beschreibungen, die mit entsprechenden sprachlichen Mitteln versuchen, die abgebildete Szene zum Leben zu erwecken:

(23) Ein heißer Wind rauscht durch das Schilf, schwüle Mittagshitze lastet über dem Gebüsch. Im feuchten Grund hat sich eine bocksbeinige Gestalt verkrochen, schwermütige Töne auf ihrer Flöte übers Land sendend. Es ist Pan, der Gott der Hirten. (Arnold Böcklin, „Pan im Schilf“) 
Zum anderen wird durch die Audioguides auf vermutete Wissensdefizite des Hörers Bezug genommen, die es zu füllen gilt, um die Kunstrezeption zu steuern. Das ist ja die primäre Funktion dieser Textsorte (s. o. Abschnitt 2). Dass der Kunstbetrachter in den Audioguides als ein „bisschen dumm“ dargestellt wird, der mit der Betrachtung des Bildes „leicht überfordert“ ist (Hausendorf/Müller 2015), wurde bereits erwähnt; dass dieser Kunstbetrachter auch wenig weiß, klingt immer wieder deutlicher durch (vgl. (24) und (25)) und noch deutlicher in Audioguides für Kinder bzw. Jugendliche wie in (26) - hier wird eine sehr naive Bildinterpretation präsupponiert.

(24) Dieser Gedanke, dass nun die Ehe auch die Zusammengehörigkeit beinhaltet, der wird durch ein ganz wunderbares kleines Detail deutlich gemacht und der damalige Betrachter hätte sofort erkannt, worum es geht, nämlich beide haben sich eingefunden in einer Laube, einer Geißblattlaube, an Staketen hinter beiden wächst dieser Geißblatt und diese Pflanze ist im Volksmund unter dem Namen ,je länger, je lieber“ bekannt. (Peter Paul Rubens, „Rubens und Isabella Brant in der Geißblattlaube“)

(25) Kippenbergers Arbeiten sind sehr vielschichtig und erschließen sich erst, wenn man sein kompliziertes Verweisgeflecht kennt. (Martin Kippenberger, „Vögel und Panzer“)

(26) Dieses Bild ist ganz gewiss kein normales Portrait. Und keine Sorge: Die Frau, die der spanische Künstler Pablo Picasso dargestellt hat, hat in Wirklichkeit bestimmt nicht so ausgesehen. Mit einem Auge im Kopf, dem anderen in der Hutkrempe, einer Nase, die man von der Seite sieht, gleichzeitig aber die Nasenlöcher von unten. Picasso hat sich in diesem Bild auch nicht über eine Dame lustig gemacht oder wollte sie verärgern. Ganz im Gegenteil: Die hier Dargestellte ist Françoise Gilot. Sie lebte für viele Jahre mit Picasso zusammen. Die beiden wurden Eltern von einem Buben und einem Mädchen. Aber warum hat der Künstler seine Lebensgefährtin dann so deformiert dargestellt? (Pablo Picasso, „Frau mit grünem Hut“)

\subsection{Dialog-Inszenierungen}

In den Audioguides gibt es grundsätzlich Kommunikation verschiedener, oben genauer konturierter Sprecherrollen mit einem Hörer, der die Betrachterrolle innehat. Diese Kommunikation wird häufig durch unterschiedliche sprachliche Strategien dialogisch inszeniert. Dies ist ein ganz wesentlicher Unterschied zwischen Audioguides und schriftlichen Museumstexten. $\mathrm{Zu}$ diesen Dialoginszenierungen gehören als explizite Strategien mehr oder weniger direkte Aufforderungen (wie „Schauen Sie sich einmal die Arme des Vaters näher an“ oder „Wenn Sie sich dann anschauen, wie etwa der Spitzenkragen gemalt ist“) sowie Fragen (wie (27) bis (31)) und das hörerinkludierende wir (wie in (11)).

(27) Fällt Ihnen an der Darstellung der Büglerin von Edgar Degas etwas auf? Ihre Arme sind jeweils in zwei verschiedenen Positionen wiedergegeben. (Edgar Degas, „Die Büglerin“) 
(28) Was machen die beiden jungen Frauen auf diesem Bild? [...] Was hat die Frau mit Hut vor: Ist sie soeben angekommen oder will sie auf Reisen gehen und jemanden treffen - vielleicht den Absender des Briefes? (Moritz von Schwind, „Der Besuch“)

(29) Der weite Wolkenhimmel nimmt fast zwei Drittel des Bildes ein, doch er wirkt drückend, grau und schwer. Doch womit sind eigentlich die Leute auf diesem Gemälde beschäftigt? (Joseph Wenglein, „Kalksteinsammlerinnen im Isarbett“)

(30) Dieser Bildzyklus spielt mit Klischees und hinterfragt auf ironische Art naive Vorstellungen von Gut und Böse. Auch kommt einem bei der „Arbeit ohne Titel - Vögel und Panzer“ die Redewendung „Mit Kanonenrohren auf Spatzen schießen“ in den Sinn. Sehen die Vögel dieses Bildes nicht wie Spatzen aus, auf die mit gelber Farbe geschossen wurde, die immer noch von ihnen runtertropft? (Martin Kippenberger, „Vögel und Panzer“)

(31) Ist dies Albrecht Dürer, wie es der Titel besagt, oder ein Bildnis Christi? Vielleicht stellen auch Sie sich diese Frage im Anblick des sanft leuchtenden Antlitzes vor dunklem Hintergrund. (Albrecht Dürer, „Selbstbildnis im Pelzrock“)

Während die auffordernden Passagen im Wesentlichen den Rezeptionsprozess steuern und die Orientierung der Bild-Wahrnehmung synchronisieren, haben die Fragen, die strukturell alle einen Dialog suggerieren, im einzelnen unterschiedliche Funktionen: Direkte Fragen wie (27) wollen die Wahrnehmung und die Bildinterpretation leiten (wobei die gewünschte Antwort und damit die dargebotene Interpretation gleich nachgeliefert wird), in (28) bleiben die Fragen, die sich hier allerdings auf die Geschichte der abgebildeten Personen beziehen, im Raum stehen. Auch (29) will mit einer durch eigentlich als (sub-)themenwechselnd gekennzeichneten - Frage die Wahrnehmung und Deutung des Bildes auf einen bestimmten Aspekt fokussieren. Die rhetorische Frage in (30) suggeriert bzw. impliziert eine bestimmte Interpretation und passt sich damit gut in die generelle Struktur der Audioguides ein, die ihre Bildbeschreibungen, Deutungen und Wertungen im allgemeinen als gesichertes Wissen präsentieren, nicht hinterfragen und auch keine alternativen Deutungsmöglichkeiten anbieten. Eine interessante Ausnahme stellt hier Beleg (31) dar, in dem eine echte Frage gestellt wird - welche allerdings - wie man im weiteren Verlauf des Textes erfährt - auch im kunsthistorischen und kunstwissenschaftlichen Diskurs unbeantwortet bleibt.

\section{Abschließende Bemerkungen}

Audioguides übernehmen heutzutage eine sehr wichtige Rolle bei der Vermittlung von kunstbezogenem Wissen, bei der Deutung und Erläuterung von Kunst im öffentlichen Raum, damit auch bei der Konstituierung und Zuschreibung von Kunst- und Sinnhaftigkeit und bei der Etablierung von Interpretations- und Deutungsweisen. Sie nutzen dabei im Vergleich zu traditionellen Lesetexten (wie etwa Museumska- 
talogen, Wandtexten in Museen etc.) eine deutlich größere Vielfalt von Strategien und Vertextungsmustern. Gleichzeitig heben sie das ,Schweigen“ der Kunstwerke auf, machen den Museumsbesuch auch zu einem Sprachereignis. Das bietet viele Möglichkeiten intensiverer und genauerer, vielleicht auch ökonomischerer Rezeption. Es birgt gleichzeitig die Gefahr der vorschnellen Vereindeutigung der Interpretation, des schnellen scheinbaren „Wiedererkennens“ oder auch der stark gelenkten, selektiven Orientierung im Bild. Letztlich können sich Audioguides - wie alle Kunstkommunikation - dem Grundproblem der ,Sprachlosigkeit‘ von Kunst und dem Problem der ,Unsagbarkeit‘ des Künstlerischen nicht entziehen, müssen hier doch Übersetzungsund Umsetzungsversuche unternommen werden, die selektiv und auch didaktisch sind. Interessant bliebe, anhand größerer, auch vergleichend angelegter Korpora zu untersuchen, wie fest und geschlossen die Erläuterungen und Deutungsangebote sind, wie sehr Fragen der Kunsthaftigkeit, unterschiedliche Wahrnehmungsweisen und Deutungsmöglichkeiten selbst thematisiert und eventuell auch problematisiert werden, statt diese als auf bestimmte Weise gegeben zu setzen. Nicht zuletzt bauen Audioguides, wie erwähnt, auf bestimmte auch kulturell geprägte musterhafte Sehund Rezeptionsweisen sowie institutionelle Konstellationen auf, die keineswegs universal sind. Daher wären auch vergleichend angelegte Untersuchungen ähnlicher Texte in anderen Sprach- und Kulturkontexten spannend. Hier eröffnet sich ein vielversprechendes interdisziplinäres Forschungsfeld.

\section{Literaturverzeichnis}

Dawid, Evelyn/Robert Schlesinger (2002): Texte in Museen und Ausstellungen. Ein Praxisleitfaden. Bielefeld.

Fandrych, Christian/Maria Thurmair (2010): Orientierung im Kulturraum: Reiseführertexte und Audio-Guides. In: Marcella Costa/Bernd Müller-Jaquier (Hg.): Deutschland als fremde Kultur: Vermittlungsverfahren in Touristenführungen. München, 163-188.

Fandrych, Christian/Maria Thurmair (2011): Textsorten im Deutschen. Linguistische Analysen aus sprachdidaktischer Sicht. Tübingen.

Gehlen, Arnold (1965): Zeit-Bilder. Zur Soziologie und Ästhetik der Modernen Malerei. 2. Aufl. Frankfurt a. M.

Gutenberg, Norbert (2000): Mündlich realisierte schriftkonstituierte Textsorten. In: Klaus Brinker et al. (Hg.): Text- und Gesprächslinguistik. Ein internationales Handbuch. Berlin u. a., 574-587.

Hausendorf, Heiko (2005): Die Kunst des Sprechens über Kunst - zur Linguistik einer riskanten Kommunikationspraxis. In: Peter Klotz/Christine Lubkoll (Hg.): Beschreibend wahrnehmen wahrnehmend beschreiben. Freiburg i. Br. u. a., 99-134.

Hausendorf, Heiko (2006): Gibt es eine Sprache der Kunstkommunikation? Linguistische Zugangsweisen zu einer interdisziplinären Thematik. In: Paragrana 15, 65-98.

Hausendorf, Heiko (2011): Kunstkommunikation. In: Stephan Habscheid (Hg.): Textsorten, Handlungsmuster, Oberflächen: Linguistische Typologien der Kommunikation. Berlin, 509-535. 
Hausendorf, Heiko (2014): je länger man hinschaut - Der Betrachter ist im Audioguide. In: Heike Roll/Constanze Spieß (Hg.): Kunst durch Sprache - Sprache durch Kunst. Duisburg (Osnabrücker Beiträge zur Sprachtheorie, 84 ), 37-56.

Hausendorf, Heiko/Marcus Müller (2015): Sprache in der Kunstkommunikation. In: Ekkehard Felder/ Andreas Gardt (Hg.): Handbuch Sprache und Wissen. Berlin (Handbücher Sprachwissen, 1), 435-454.

Kunz-Ott, Hannelore (Hg.) (2012): Mit den Ohren sehen. Audioguides und Hörstationen in Museen und Ausstellungen. München.

Luhmann, Niklas (1997): Wahrnehmung und Kommunikation. Zur Reproduktion von Formen. In: Luhmann, Niklas: Die Kunst der Gesellschaft. Frankfurt a. M., 3-91.

Popp, Kathrin (2013): Das Bild zum Sprechen bringen. Eine Soziologie des Audioguides in Kunstausstellungen. Bielefeld.

Schulze, Holger/Hanna Buhl (2012): Eine Ausstellung hören? Über Notwendigkeit und Qualität von Audioguides. In: Kunz-Ott, 27-31. 